\title{
Erratum: Bose-Einstein Condensation of Long-Lifetime Polaritons in Thermal Equilibrium [Phys. Rev. Lett. 118, 016602 (2017)]
}

\author{
Yongbao Sun, Patrick Wen, Yoseob Yoon, Gangqiang Liu, Mark Steger, Loren N. Pfeiffer, \\ Ken West, David W. Snoke, and Keith A. Nelson \\ (Received 14 March 2017; published 7 April 2017)
}

DOI: 10.1103/PhysRevLett.118.149901

We have found numerical errors and typos in the main text and in Fig. 3 of this Letter. These do not affect any conclusions in the paper. The Supplemental Material has also been updated for clarity.

(a)

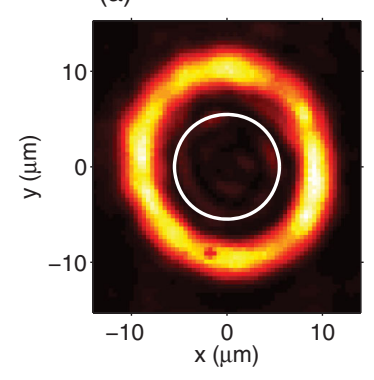

(b)

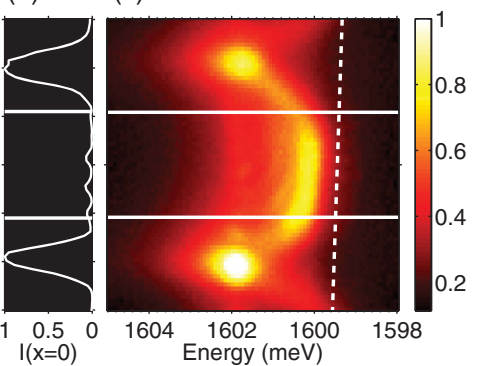

FIG. 1. (a) Reflection of the excitation beam from the sample surface. The white circle indicates the region of the sample that is observed in photoluminescence (PL) imaging measurements after spatial filtering. (b) Normalized excitation light intensity along the $x=0$ line through the center of the excitation ring pattern shown in (a). (c) Spectrally resolved PL along $x=0$. The PL within the solid white lines is collected and imaged onto the spectrometer CCD in the far-field geometry for the polariton distribution measurements. The dashed white line indicates the photon energy gradient deduced from the low-density spectrum.
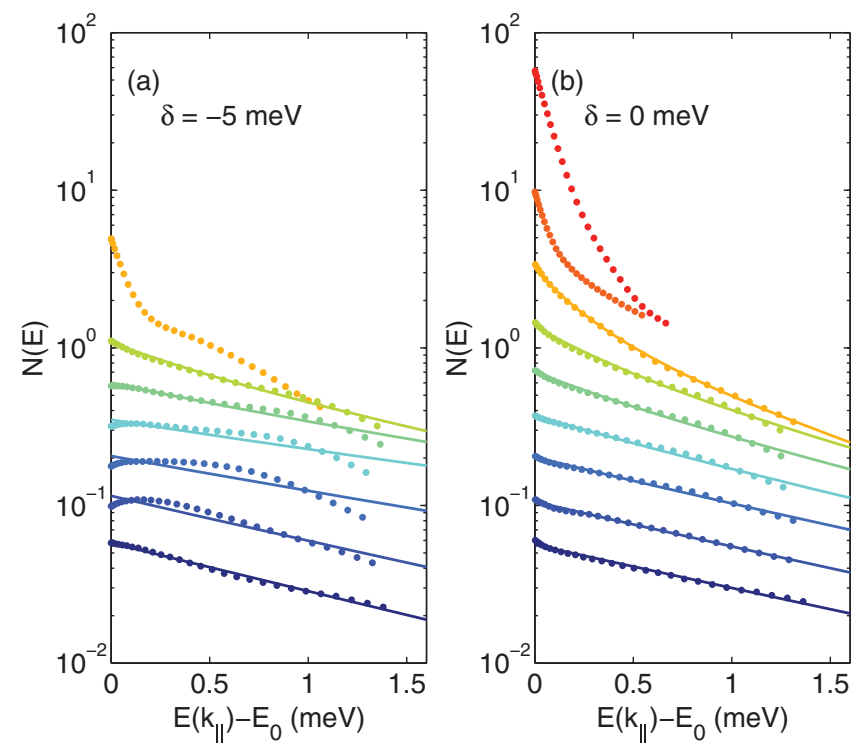

FIG. 2. Energy distributions of polaritons in the center of the trap at (a) $\delta=-5 \mathrm{meV}$ and (b) $\delta=0$ meV at a bath temperature of $T_{\text {bath }}=12.5 \mathrm{~K}$ at different pump powers (see Supplemental Material for values [32]). The solid curves are best fits to the equilibrium Bose-Einstein distribution in Eq. (1). The fitted values of $T$ and $\mu$ are shown in Fig. 3. The power values from low to high are 0.12, 0.24, $0.45,0.71,0.93,1.07,1.10,1.12$, and 1.14 times of the threshold values $P_{\mathrm{BE}}$, which are $382 \mathrm{~mW}$ and $443 \mathrm{~mW}$ for detunings $\delta=-5 \mathrm{meV}$ and $\delta=0 \mathrm{meV}$, respectively. 

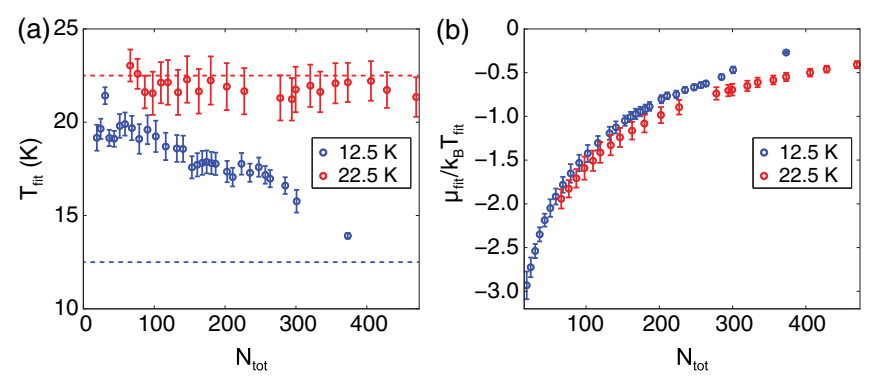

FIG. 3. (a) Effective temperatures of polaritons for bath temperatures $T=12.5 \mathrm{~K}$ (blue points) and $T=22.5 \mathrm{~K}$ (red points) at different pump powers, extracted by fitting the energy distributions [shown in Fig. 2(b) for the 12.5-K case] to the equilibrium BoseEinstein model. The dashed lines indicate the helium bath temperatures. (b) Reduced chemical potential $\alpha=\mu / k_{B} T$ for bath temperatures $T=12.5 \mathrm{~K}$ (blue points) and $T=22.5 \mathrm{~K}$ (red points) at different pump powers.
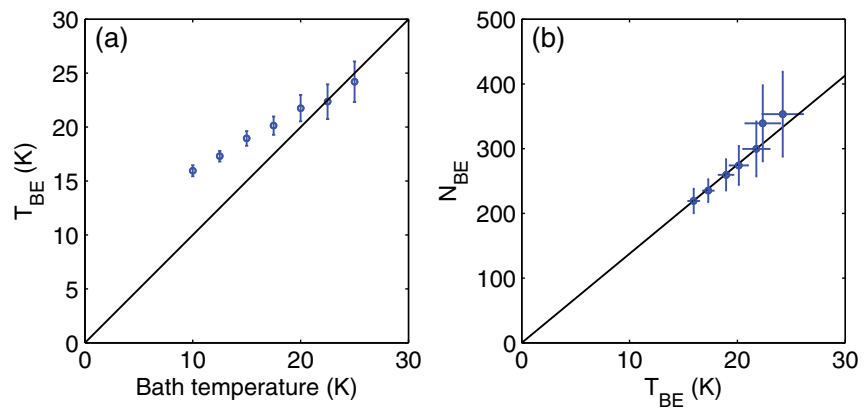

FIG. 4. (a) The critical temperature as a function of lattice temperature for $\delta=0 \mathrm{meV}$. The solid black line indicates $T_{\mathrm{BE}}=T_{\text {bath }}$. (b) Phase diagram of the transition to a degenerate Bose gas. The solid black line shows the best fit of a linear relation $N_{\mathrm{BE}} \propto T_{\mathrm{BE}}$.

In Figs. 3(a) and 3(b), we had an additional factor of two in the horizontal axes $N_{\text {tot }}$, the total number of polaritons in the trap, due to adding both positive and negative $k$ vectors in $k$-resolved data. This gave twice larger values of $N_{\text {tot }}$. This miscalculation was not done anywhere else, including the calculation of $N(E)$ in Fig. 2 and the calculation of $N_{\mathrm{BE}}$ in Fig. 4.

Other errors in the main text are as follows. (i) At the top of the right column on page 2, we noted that "the barrier height is varying by $1 \mathrm{meV}$ from one side to the other." However, the variation in the barrier height was less than $0.2 \mathrm{meV}$ along the ring. (ii) Two lines below, we stated that "the barrier is slightly wider than the laser profile because excitons propagate up to $10 \mu \mathrm{m}$." However, the excitons diffuse only up to $5 \mu \mathrm{m}$ as shown in Fig. 1(c). Similar measurements discussed in the Supplemental Material of Ref. [52] also confirm that excitons do not propagate further than $5 \mu \mathrm{m}$. (iii) In the middle of the right column on page 2, we described $N(E)$ as "the number of polaritons." Although this is not incorrect, we emphasize that this refers to the occupation number per quantum state, not the total number of polaritons in the trap $N_{\text {tot }}$. (iv) In the caption under Fig. 2, the case $\delta=0 \mathrm{meV}$ is described as "strong interactions, nonequilibrium." This should be "strong interactions, equilibrium." Also, at the end of the caption, " $\delta=5 \mathrm{meV}$ " should be " $\delta=-5 \mathrm{meV}$." (v) In the caption under Fig. $3, N_{\text {tot }}$ refers to the number of polaritons within the entire trap area of $\pi(9.5 \mu \mathrm{m})^{2}$. Similarly, $N_{\mathrm{BE}}$ in the phase diagram of Fig. 4(b) refers to the critical number of polaritons within the entire trap area of $\pi(9.5 \mu \mathrm{m})^{2}$. 